\title{
User-centred design vs. "good" database design principles: a case study, creating knowledge repositories for indigenous Australians
}

Natalya Godbold

University of Technology, Sydney. Email: ngodbold@gmail.com

\begin{abstract}
In 2006-07, the author was involved in the redesign of a database storing traditional knowledge of Australian Aboriginal clans. The project raised issues relating to the design of repositories for indigenous knowledge, including theoretical approaches taken to ontology (knowledge structures, classification systems) and metadata creation. This paper describes the design process where user centred design principles accommodated an emergent contrast between traditional knowledge customs and "good" database design principles. Theoretical approaches taken to ontology are presented, and the format of the restructured database is discussed in general terms. Based on evidence from usability testing and qualitative research, it is concluded that a flexible ontological system was needed to provide the customisability required by indigenous users - a system whose incarnations may usefully represent widely differing aboriginal cultures.
\end{abstract}

\section{Background}

Traditional Knowledge Revival Pathways ${ }^{1}$ (TKRP) was initiated in 2001 by elders of the Kuku Thaypan clan from Cape York in the north-eastern corner of Australia, who were concerned that the cultural knowledge of their clan would be lost when they passed on. Victor Steffensen, a Tukaluk man who had been learning from the Kuku Thaypan elders for many years, began at their request to record the elders on video explaining their knowledge, beliefs and practices for future use by their clan.

All the knowledge the old people taught me over ten years, I want to pay the old people back by showing the young people.

Victor Steffensen

Steffensen built a flat-file database using Filemaker Pro to store the video footage. By late 2005 the call was made to redesign the flat-file database into relational form before the project became too large and use of the database became widespread. In February 2006 in close consultation with Steffensen and Andrew Wood from DSTC, the author undertook the redesign project with the aim of creating a robust, flexible, searchable infrastructure to support the archiving and ongoing use of the traditional knowledge being gathered by indigenous community members, while retaining the look and feel and browsing structure presented in the original database. The database redesign project took place over a year and a half until June 2007.

It is important to emphasise that what was being designed was a database shell, an empty database, which would be passed to participating clans to independently record their own traditional knowledge in the context of their unique knowledge system. Replicas of the generic original database would be taken into unknown and far flung environments. There was no 
requirement to ever join the individual replications back together again. Clans need to be able to document their knowledge while maintaining cultural duties required of them such as secrecy and custodianship of knowledge, so individual databases created by each clan remain the separate property and business of the clan. The importance of maintaining separation between different knowledge of different clans is taken literally by participants to the extent that on one particular site, two separate databases are being maintained to contain the knowledge of two separate language groups, reflecting traditional knowledge boundaries.

The content of individual databases by different clans is determined by the clans themselves, in particular by what their elders find to say. The database had to be made capable of storing and retrieving whatever knowledge the associated elders have to impart to their clan. Web based delivery of TKRP databases is not envisaged, and such access stands in contrast to the duties of knowledge custodianship described above.

It was established that we should work together towards a database which:

- is usable by people who mainly do not have English as a first language, and who have limited experience of computers;

- is acceptable to Aboriginal people;

- provides accessible data: the original model of the database provided no searchability beyond browsing by category;

- is appropriately secure:

○ protecting data from accidental deletion.

- able to partition knowledge within the clan using password access, for example showing women's business only to people with the women's password.

Other traditional knowledge databases have taken a sophisticated password driven approach to knowledge custodianship such as the Indigenous Knowledge Management System designed by DSTC described by Hunter, in comparison to which TKRP's approach to passwords remains a simple affair?

The project raised a host of issues relating to systems design, including theoretical approaches taken to ontology and metadata creation, which are discussed here.

\section{Principles of "good" database design}

\section{Metadata}

In a well designed database, metadata is used to support data management and workflow as well as to speed up and enrich searching. Organisations such as the Australian Information and Communications Technology in Education Committee ${ }^{3}$ and authors such as Gilliland ${ }^{4}$ recommend the use of established metadata standards and existing vocabularies to support interoperability - sharing information between separate systems. At first glance, ignorant of some of the issues described above, the TKRP project appeared to be a good candidate for creating data repositories which can later be shared. For example information from many clans could be combined to create a mega-repository of indigenous Australian knowledge. Another potential situation requiring ease of data sharing may be if clans wish to share knowledge with government departments or NGOs for the purposes of passing on land management techniques or medical knowledge. To design for interoperability, potentially useful vocabularies exist pertinent to Australian Aboriginal culture, including the AIATSIS Thesauri ${ }^{5}$. Language 
and location vocabularies such as those developed by the Australian Bureau of Statistics ${ }^{6}$ could be considered. Biological classification systems also suggest themselves as pertinent to this project, if elders wish to discuss knowledge relating to animals and plants, for example the system described by the International Association for Plant Taxonomy ${ }^{7}$. As will be discussed, attempts to make use of these tools led to difficulty.

\section{User centred design}

As described by ISO 13407 (International Organization for Standardization) user centred design mandates that designers properly comprehend and specify the detail of user requirements and the context in which a product will be used before producing solutions. Designed objects should then be evaluated against the specified requirements. Nielsen ${ }^{8}$ discusses participatory design, which focuses on collaborating with intended users throughout the design and development process, rather than designing a system "for" them". Authors including Long, Lage, \& Cronin ${ }^{9}$ describe user-centred design, in which "patrons are more likely to use a resource when they have input into its development ... the process of usercentered design builds a sense of community and support". These principles fitted the goals of the TKRP development team, being to develop a database which participants enjoy and are proud of - and for people to be enthused by the database and therefore drawn further into the TKRP project

I want to keep the heart of the project: keep the people involved in the database... the database is a living thing, flexible, developing... getting people moving on country, and connected to what's breathing out here. ...linked in to the old people, the values and the people.. [and] aligned with traditional law

Victor Steffensen

As will be described later, the principles of user centred design led the design team into direct conflict with the mandate to use interoperable metadata using established national standards. Even before that tension surfaced however, an immediate consequence of user centred design is worth pointing out. The development of "personas" is a classic step in user centred design. Based on user research, designers create descriptions of classic users, named characters with goals, needs, preferences, likes and dislikes. Discussing the development of personas, Cooper and Reimann ${ }^{10}$ note: "If no clear primary persona is evident, it could mean... the product is trying to accomplish too much." Products can't be designed for everybody. Rather, designers need to make choices about who the principal users will be. A glance at a map of Aboriginal Australia such as that produced by David Horton ${ }^{11}$ reveals several hundred language groups with all the cultural variety that implies. Designing one product for such a diverse "user group" presents as problematic, while describing the result as a database "for indigenous knowledge" as if that were a unified system, is unrealistic.

\section{The negotiation process of redesign}

..the best databases for indigenous peoples to use for their own purposes of knowledge transmission may be frustratingly difficult or counter-intuitive for western scientists... ${ }^{12}$

In line with the principles of user centred design, all changes made to the database were negotiated with TKRP. However a certain creative tension was apparent at all times during these early negotiations: databases are systems where entities are stripped down to their essential qualities, after which relationships are identified and made manifest. By nature this is a reductionist process. Yet indigenous knowledge is described as contextual, linked, and continuous. When we first sat down to thrash out the issues of database redesign, Victor 
scolded me time and time again for trying to strip items or knowledge away from context. 'That's not the right way,' he would say. He explained again and again the importance of maintaining the connection between country, knowledge, ceremony and people. But the database had to be built, so the problem of reductionism returned. As are all relational databases, the database was built with entities and relationships: fragmented and then reconnected. Database sections were presented as design solutions to Victor, who saw the operational whole and worked with that. Western reductionism happened covertly, "under the bonnet" and away from the indigenous eye. Law ${ }^{13}$ describes a process of knowledge creation whereby, once an understanding has been reached, the steps that led to that understanding can be allowed to fade into the background. The process of breaking down structures was alien to Kuku Thaypan practice; but perhaps we came out successfully with a re-connected system allowing the design process to disappear.

From Victor's perspective, some aspects of the redesign were non-negotiable, such as the browsing structure. Knowledge records could be put into the database without requiring a fixed browsing structure: without first navigating to "wallaby", one could put a wallaby video into the database. As described by authors such as Bowker and Star ${ }^{14}$ the concern was that a browsing structure useful for one clan is not likely to be a good taxonomy for all. However it appeared that the act of navigating through the categories from the front page (all creation) to the animals, to the land animals, was to trace a journey or path which paid correct homage to the wallaby knowledge and its place in the database and in the world. Navigating the browsing structure reminded me of ceremonies linking place with meaning. The journey through and recognition of knowledge structure was as satisfying as arriving at and thinking about "wallaby". So the browsing structure stayed.

Also non-negotiable were the "look and feel" and general layout of the screens - 'it looks indigenous and the old people like that.' They didn't want a database that 'looks like it came out of a university'. The same kind of requirement emerged during design of Ara Irititja : 'it can't look like something Microsoft would make' 15. A key issue to emerge for the Ara Irititja project was designing and implementing an appropriate user interface. Despite the warning, it was surprising to find the extent to which users valued the relationship between interface and cultural signifiers. There was considerable disappointment that it was not possible to find an efficient way to allow them to easily change the colour schemes for their individual databases, which would have allowed them to visually signify their ownership of the database incarnation and the knowledge therein.

\section{Ontological Flexibility}

Bowker and Star ${ }^{16}$ point out the political and ethical ramifications of classifying one knowledge system using the classifications of another, dominating culture. Christie ${ }^{17}$ specifies the problem in terms of database design for Yolngu knowledge, noting (with reference to Agrawal'18) that for a database to be indigenous, "its architecture and structure, its search processes and interfaces, its ownership and uses must reflect and support context specific indigenous ways of being and knowing, and people's control over their own knowledge". In particular, a digital repository for indigenous knowledge which forces the user to use western, curatorial, or other culturally mismatched taxonomies limits the capacity to properly categorise and contextualise indigenous data ${ }^{19}$. And in fact, representatives of TKRP clearly expressed their rejection of pre-designed thesauri or taxonomies. They expressed distaste both for the intrusion of "western" ontologies and the need to juggle and comprehend great lists of terms. 
The original flat-file database in use by TKRP was built on a number of static classification schemes which were hardwired into the architecture, the standout being a system of seven main knowledge categories (for example "animals" and "plants" were two such categories). Some categories then had built in subcategories, for example, the category "animals" had subcategories including "land animals", "birds" and "frogs". The knowledge categories were used to create the browsing structure mentioned earlier. A participant with video footage on the subject of the wallaby, commencing on the home page, would navigate into "animals" and then into the subcategory "land animals", where a record to hold the footage for wallaby could then be placed.

Although this knowledge structure or classification system was indigenous in origin, it was also local to the Kuku Thaypan people of far north Queensland, whose rainforest culture was recognisable in categories such as "frogs". One immediate goal of the TKRP project was to expand its participant base; a clan in Western Australia, for example, was poised to begin a database of their own, and another in the south east of the continent. At the time of writing, a group in New Zealand have commenced using the database to store their traditional knowledge. Perhaps a system was needed which would allow individual clans to generate their own knowledge categories: a browsing category "frogs" was unlikely to be regularly useful to a desert dweller, while other culturally important categories, by definition not able to be predicted by outsiders, may be missing. One simple way to introduce flexibility here was to allow users to rename categories: if before a clan begins to use their database it is obvious that "frogs" is unlikely to be needed much, it could be renamed and used for "feral animals" for example. Given the very short turnaround time for the release of version 2.0 , the relational database was structured to allow categories to be renamed in theory, but the coding was not written in time for version 2.0 to enable renaming categories in practice. Usability testing of version 2.0 revealed misgivings in some quarters with the applicability of all the knowledge categories 'There wasn't enough categories for us.. that was made for Laura people.' On this evidence, the work required to allow renaming of categories was completed in version 2.4 , and coding was added to allow users to add or remove plant and animal subcategories, providing a range of four to six subcategories which can be named as deemed fit.

The result is a partially flexible, partially re-nameable classification system which doubles as the browsing structure for the database. Notably, we have not completely removed hierarchy to provide what Verran et al recommended 20 : a flat, clear space encoding no assumptions (through metadata) about the nature of the world or the nature of knowledge, on which participants can build the topology of their world. The database is still built over a rigid browsing structure of navigation points: seven categories which can be renamed, some containing subcategories, of which two sets can be renamed and the number of subcategories modified. An important aspect is likely to be that categories can be translated into indigenous names.

\section{Structural entities}

At a fundamental level, entities in the database were split into two categories: knowledge and tags. Tags are used to tag knowledge, indicating that the knowledge is "about" or "concerns" that tag. Tags include stuff or things - anything in the universe about which you might have knowledge: places, people, animals, plants, spirits. Tags are also used to house a list of facets identified by Victor in the original database: craft uses, food uses, etc... these facets loosely describe types of knowledge. There is a set list of them, to which the user can add more. All tags are classified into a subcategory, and therefore placed into the knowledge and browsing schema, as shown at Figure 1 below. Knowledge records (videos, reports, documents, pictures) can be linked to an infinite number of tags. 


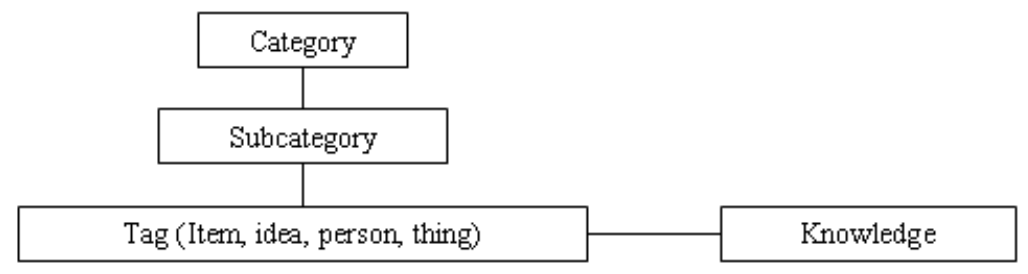

Figure 1. Five main tables (entities) used to create a flexible structure. For example, Category "Plant" may include subcategory "grasses" which may include tag "speargrass". Knowledge about "speargrass" may be a video showing how to prepare speargrass for craft uses.

\section{Metadata Strategies}

During initial meetings with TKRP, it was established that pre-designed thesauri or taxonomies are inappropriate for participants, who would resent both the intrusion of "western" ontologies and the need to juggle and comprehend great lists of terms.

The tagging system described above, where knowledge is linked to tags, represented a compromise. Yet the real ontological freedom provided by the database emerges from this capacity to link any video to any object in the database. When a video segment is entered into the database, the user links it to ideas, objects, animals, ceremonies or any other entity they have chosen to put into the system. It is envisaged that because users name all the tags in the system and choose how knowledge is to be tagged, each database will develop a knowledge network representative of the clan using it. The compromise is that the improvement in customisability is paid for by databases with divergent metadata, an issue which will be discussed later.

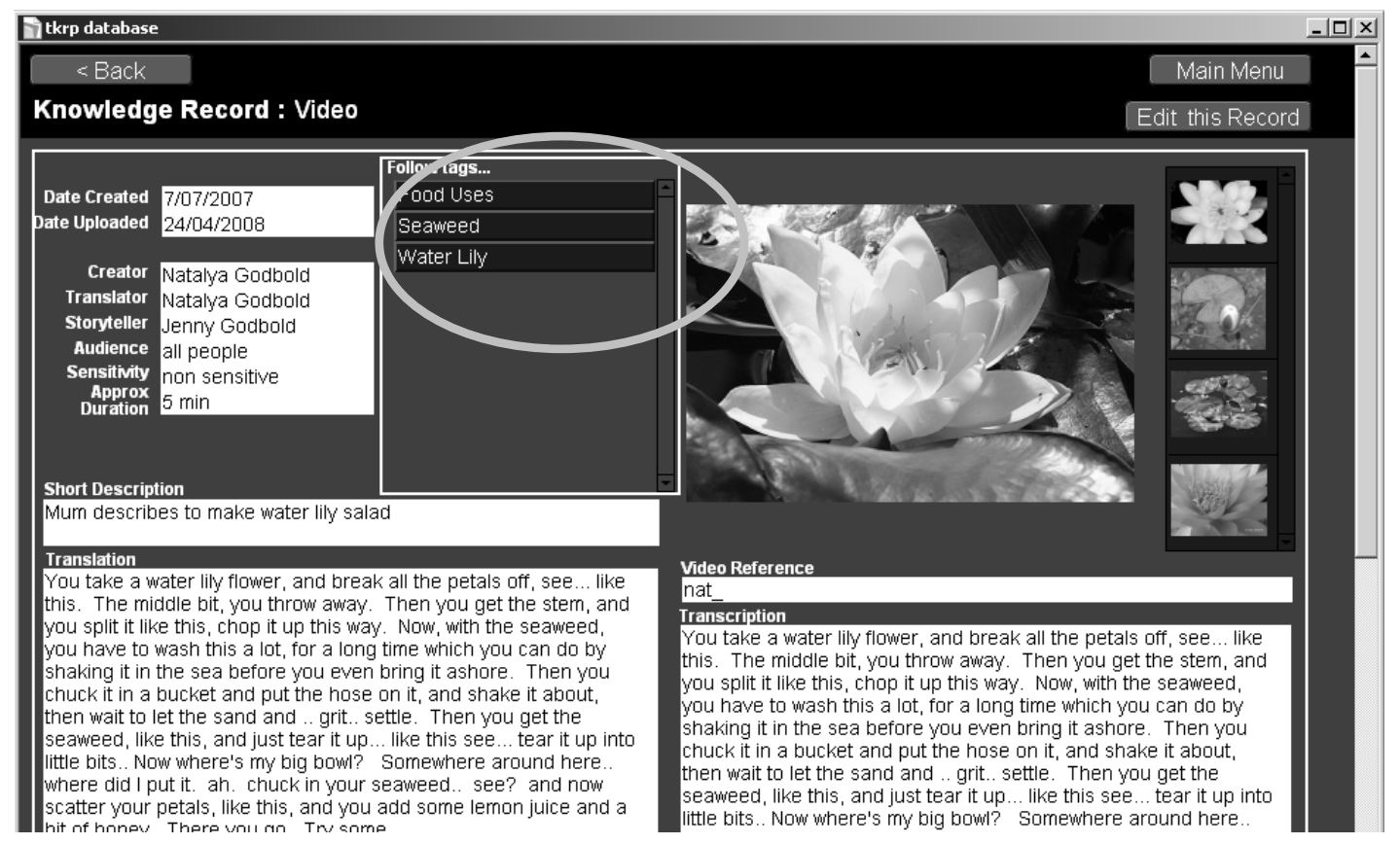

Figure 2. Sample knowledge record, showing clickable links to "food uses", "seaweed" and "WaterLily"

When browsing, the user can move along links from knowledge to item to knowledge (or they can use the search functions described below). A mock up of a video record is shown at Figure 2 above. The video is imagined to be about making a salad out of seaweed and water lily, a recipe invented by the author as an example only. Three links are visible (red buttons, circled), 
marking the video as being "about" Food Uses, Seaweed and Water Lily. The user can navigate through the database by clicking on these buttons, for example clicking on the red Water Lily button takes the user back to the Water Lily record.

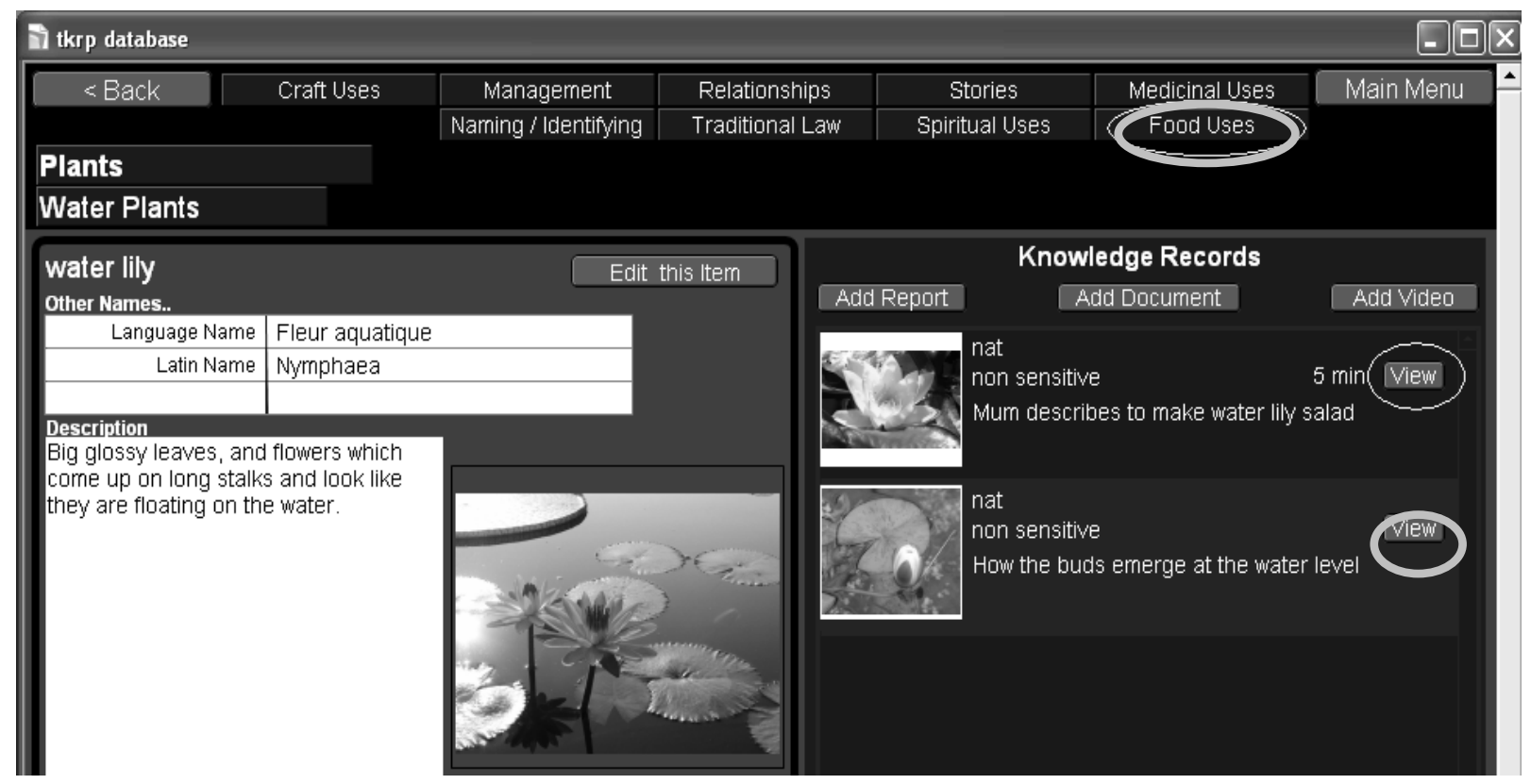

Figure 3: Sample plant record: Water Lily.

From the Water Lily record, shown at Figure 3 above, the user could navigate again, for example clicking on the food uses button at this screen will initiate a search for food uses of the Water Lily, while clicking on a knowledge record (listed down the right hand side) will take the user to the chosen knowledge record, such as the second example shown: a video in which the emergence of water lily buds is described.

As Mathe notes:

systems employing free-form tagging ... encouraging users to organize information in their own ways, are supremely responsive to user needs and vocabularies, and involve the users of information actively in the organizational system ${ }^{21}$.

The system of tagging proposed here is not completely "free-form" in the manner associated with applications such as Flickr, where each individual user makes their own tags as they go. Rather, in a manner inspired by Guy et al22, the user is first presented with a list of tags which already exist in the database, and encouraged to choose from them. If no pre-existing tags are appropriate, the user can then create a new tag. It is hoped that this small effort involved and the imperative of first viewing existing tags will reduce the creation of synonym tags. The proposed system is therefore a combination of hierarchical classification and controlled tagging.

The tagging system of metadata was in place in time for version 2.0, and included in the usability testing conducted on that version. Once they had been shown that the system of linking videos to tags was there, users embraced it with great enthusiasm, describing it as a simple system. Further usability testing of version 2.4 would reveal if users have continued to use the linking system in the long term. 


\section{Public Access}

In the bigger picture for TKRP is recognition that much of the knowledge to be recorded has value to a far broader community than the clans owning the knowledge.

[I want to] do something for aboriginal people and non aboriginal people. Recognising traditional knowledge and having aboriginal people recognised as people with a role in society, a contribution

(participant)

Therefore the following projects are in progress:

- negotiating access by Government agencies to land management, fire management and water management information. Traditional knowledge already informs a new style of land management occurring in Cape York. Specifically, fire management knowledge is being used as part of the joint management of Lakefield National Park by the National Parks and Wildlife Service (NPWS) and the Kuku Thaypan clan

- planning for the creation of an "Education shell" version of the database, containing selected, combined information from different communities, for use by schoolchildren around Australia. The Education shell provides the possibility of a combined database of shared knowledge, and could be a rich archive of cultural, historical, and ethnobotanical information from an Aboriginal Australian perspecitve

The current project was the necessary first building block for public databases such as these. Before traditional information can be accessed by the wider public, it must be gathered in a systematic way.

There is an obvious contradiction here between the goals of public access to selected information, and the cultural mandate of not sharing or releasing traditional knowledge. The contradiction affects the project in very concrete ways.

Firstly, if knowledge is to be easily moved into other databases, then there needs to be a perfect match between data structures of the private and public repositories. As soon as the data structures differ, someone is required to pick over the data and adjust it to fit. This person then has to make decisions about how records should be reclassified - sometimes decisions they cannot make, or which would have been made differently by a person from the originating clan. The options are, force each database to have a fixed structure and to use pre-defined classification schemas, or face a lot of work when the time comes to merge records from the differently divergent database variations.

Second, if a private clan-based database is ever destined to send records to a public repository, then all of it could potentially be so treated, raising issues of trust and security for knowledge custodianship. Some elders already approach the TKRP project with suspicion:

they're thinking that all the stuff getting stored in and all its gonna be exposed to everybody.

(participant)

Although the politics of providing indigenous knowledge to the wider community were not discussed in depth at the time, Agrawal makes many interesting points on the issue, raising questions about the "validation" of indigenous knowledge by science and suggesting that once 
the knowledge systems of indigenous peoples are separated from them and saved, there is little reason to pay much attention to indigenous people themselves - a process set to perpetuate asymmetrical relations of power and poverty 23 .

As it is fundamental to the design of the database, some of these issues were raised with TKRP before any redesign began. It was established by project participants that the ability of users to "just get the data in" was of higher short-term priority than consistency of entries across different shells. For the sake of such immediate usability, a set of sacrifices were made ending in the decision to allow customisability of metadata, honouring the differing ontologies of participating clans. As time goes by each clan will develop their own tags and category labels, creating a set of structures which are congruent in form, but divergent in content. Each database will morph into a network and potentially also an ontology of its own. If merged, the super-set of all tags and category labels would need to be inspected and links reformed by hand in what is likely to be a heinously messy process.

Interestingly, the fact that they are likely to be difficult to rejoin was seen as a design strength, as it emphasised traditional duties of knowledge custodianship and the separation of knowledge between clans and between other divisions in indigenous society.

\section{Other architecture and design issues}

\section{Search Strategies}

The flat-file database had no search screen, and items could only be accessed by browsing in likely places. A single line search field was included in version 2.0, and version 2.4 of the database the search screen allows users to search for either items or knowledge, and to display all items or display all knowledge. During usability testing, a participant was noted using the display all button with satisfaction:

See everything I've done. Show em' all.. Look, look.. All that knowledge.. That's been recorded...

(participant)

Search facilities use scripting to allow the user to enter part of the search string they require. If for instance, they enter "wallab", the system will search for anything with the string "wallab" in it, and return both "brush wallaby" and "wallaby grass". The search for the given string is conducted throughout most fields of the record - so a search on a clan name finds the clan, people in the clan, and country associated with the clan. Results can then be sorted by category or alphabetically.

When presenting search results, and during the processes of browsing, thumbnails are used to supplement words as much as possible, to facilitate non-language based use.

\section{Browsing strategies}

The existing system was already strong in browsing mechanisms, all of which were retained and enhanced in the new database.

In addition to the formal browsing structure, the user can now browse by moving along links from knowledge to item to knowledge - or they can navigate via the search screen. 
"Breadcrumbs" at the top of any page indicate where the user "is" and allows them to click on and move back to other places. For instance, if you are looking at a ti tree record, the "breadcrumb" may read "Plants - Trees - Ti Tree". The user could click on "Trees" to go back to a list of all trees in the database, or on "Plants" to inspect the data on all plants.

\section{Scalability}

Hunter ${ }^{24}$ notes that systems such as FileMaker Pro are not suitable for large IKM databases which can reach hundreds of thousands of records. Individual TKRP databases are likely to be on a more modest scale (hundreds of records), and involve a single user at a time.

FilemakerPro reports an 8 terabyte limit per database depending on factors such as disk space and $\mathrm{RAM}^{25}$. As the product was already in use by TKRP and is likely to support the scale of the project, project stakeholders agreed to continue with Filemaker.

\section{Interoperability}

The current project requires a database which can be distributed as an easily accessible data gathering shell for users on country. As has already been noted, interoperable metadata is not a requirement as these shells are never intended to be merged in their current form, nor to be publicly accessible. In fact as noted above, the TKRP database in any of its in-community incarnations is a small scale, private, local database which will protect itself from data sharing by the extent that it has morphed via customisation of category names and use of indigenous language to name items.

\section{Data Entry Strategies}

To control data entry and reduce incidence of issues such as incorrect spelling, accidental multiple entries and loss of data due to literacy difficulties ("too hard to type" etc), the following strategies are used:

- iterative drop down lists - vocabulary used in other tables (such as lists of people's names) appear in drop down lists from which the user selects an entry

- spelling checker - it is noted that participants enjoy adding traditional language terms; Good it's learnin' my language.. database now...

- defaults completing most fields automatically, which are modifiable and preset by users;

- background scripts to detect and create relevant tags (e.g. if the user enters a new video segment from the grass wallaby record, the video will automatically be tagged as belonging to the Grass Wallaby);

- systems to control data entry (check for integrity, completeness, possible duplication and prompt if necessary).

\section{Discussion}

The fact that categories can be renamed means that the browsing structure could be presented mainly using indigenous terms. Tags could also be given Aboriginal language names (perhaps with the English translations as alternative names), producing a database presenting strongly in the indigenous language.

It will be interesting to see if future mutations of the database do take the opportunity to rename their browsing categories, and to what extent they do so, and how successfully the new names sit over the old categories and subcategories. 
Following the completion of version 2.4 and having given it time to settle into use, a number of questions emerge:

1. How do participants view version 2.4? With pride (as per the goals of the project)? With frustration? Has it been abandoned and why? How many users are out there? In what geographical locations?

2. Security, password, secret knowledge issues - are they treated too lightly in this system?

3. Is the TKRP project, in whatever guise it currently takes, participating in the process of creating a repository of local knowledge for the interest of that community - and in their interests?

4. What sorts of ontologies are being created? How usefully do participants feel they are able to model the knowledge system of their own culture? Do they need more categories? Do they need more control over the topology of the browsing structure?

5. In addition to the formal browsing structure, the user can now browse by moving along links from knowledge to item to knowledge, or they can navigate via the search screen. Is this a subversion of the journey reinforced by the browsing structure which acknowledged relationships between knowledge and knowledge structure?

6. Perhaps because there are many indigenous cultures, and they are small and diverse, they are grouped together and attempts like this are made to design systems "for indigenous culture" which will be useful to "them all". To what extent can design succeed in transcending culture and ontology, creating a database useful to "all cultures"? Could my father, for example, use a TKRP database shell to record our family's stories of my English, Irish, Chinese and French ancestors, and his knowledge of seafaring?

These are questions which could be taken up by future research. Specifically, Hughes \& Dallwitz [26, p. 157] suggest that a solution to many of the problems they encountered would be to have more indigenous peoples providing IT for themselves... nor are they the only authors to make this evident suggestion. The TKRP database in its original version was created by an indigenous person - Victor Steffensen - and during testing of version 2.0, indigenous participants both demonstrated IT skills and commented on how the project extends their involvement with computers. One jokingly suggested he might '..build my own database [loud laughter] that'd be scary wouldn't it!'. If a project like TKRP had such an outcome it would benefit all concerned.

\section{Conclusion}

This project presented a couple of fundamental puzzles. The first was that the database must be immensely usable and culturally acceptable to individual clans, whose needs and cultural norms are by definition, different. To this end, the database must be robust enough to support inexperienced users and obtain consistent data entry, for which designers would normally prescribe drop down lists of pre-set metadata - yet able to take "any data the Elders choose to talk about", mandating flexible, user-definable metadata - by users who are not all computer literate, nor receptive to western defined thesauri and classification systems.

The second was that while the database was designed to be a private, local solution for individual communities, there has always been discussion of projects such as the creation of public access databases for various purposes, with all the political and cultural processes that implies. If such databases eventuate, source data from the databases currently in design 
should be able to be easily merged, again implying the need for solid, consistently applied metadata using nationally or internationally established standard vocabularies.

A solution which will cover all these requirements has not been found. The project has gone in the direction of a product which by virtue of a degree of flexibility in its ontological structures, may be useful to widely differing aboriginal cultures. By doing so we are led away from data which could be easily merged into public or common databases. That the merging of mutated databases would be a difficult process emerges strangely as a design strength. It may encourage communities to trust their data to a TKRP database, since further (re)use of data would be a conscious and appropriately painstaking operation.

\section{Acknowledgements}

The author is grateful to Victor Steffensen and Andrew Wood, for the opportunity to be involved in this inspiring project, and to my Masters supervisors Jan Houghton and Maureen Henninger, as well as Jacqueline Gothe for their guidance, criticism and encouragement. It was an honour to witness appropriate examples of the culture, and meet representatives of the Kuku Thaypan, Kuku Yalanji and Aurukun people.

TKRP has been funded and/or supported at different times by Balkanu, NHT, Envirofund, Cape York Peninsula Development Association, CRC Tropical Savannas Management, The Christensen Fund, University of California, James Cook University, ACF, University of Technology - Sydney, NAEA, Indigenous Enterprise Partnerships, Telstra, the Distributed Systems Technology Centre (DSTC) and Questacon.

\section{NOTES}

1 TKRP Traditional Knowledge Revival Pathways 2009 at http://tkrp.com.au/index.php

$2 \mathrm{~J}$ Hunter 'The Role of Information Technologies in Indigenous Knowledge Management'. Australian Academic and Research Libraries (AARL), v36 n2 2005 pp 109-125.

${ }^{3}$ Australian Information and Communications Technology in Education Committee About technical standards 2007 at http://www.aictec.edu.au/aictec/go/standards

${ }^{4}$ A J Gilliland Setting the Stage. In M Baca (Ed) Introduction to metadata: pathways to digital information $3^{\text {rd }}$ ed Los Angeles CA Getty Publications 2008.

${ }^{5}$ Australian Institute of Aboriginal and Torres Straight Islander Studies (AIATSIS) AIATSIS Thesauri 2008 at http://www1.aiatsis.gov.au/thesaurus/

${ }^{6}$ Australian Bureau of Statistics 1291.0 - A Guide to Major ABS Classifications 1996 at http://www.abs.gov.au/AUSSTATS/abs@.nsf/Lookup/1291.0Main+Features11998?Op enDocument

7 International Association for Plant Taxonomy International Code of Botanical Nomenclature (Vienna Code) 2005 at http://ibot.sav.sk/icbn/main.htm

8 J Nielsen Usability Engineering. Boston Academic Press 1993 p 88. 
${ }^{9} \mathrm{H}$ Long K Lage \& C Cronin The flight plan of a digital initiatives project, part 2: Usability testing in the context of user-centered design OCLC Systems \& Services 21(4) 2005 pp 324-345.

${ }^{10}$ A Cooper \& R Reimann About face 2.0 : the essentials of interaction design Indianapolis Wiley $2003 p 72$.

11 D Horton Aboriginal Australia

12 M Christie Computer Databases and Aboriginal Knowledge Learning Communities: International Journal of Learning in Social Context v1 2003 pp 4-12.

$13 \mathrm{~J}$ Law After method : mess in social science research (1st ed) New York Routledge $2004 \mathrm{p}$ 20.

${ }^{14} \mathrm{G}$ C Bowker \& S L Star Sorting things out : classification and its consequences Cambridge MA MIT Press 1999.

15 M Hughes \& J Dallwitz Ara Irititja: Towards Culturally Appropriate IT Best Practice in Remote Indigenous Australia. In L Evelyn Dyson M Hendriks \& S Grant (Eds) Information technology and indigenous people Hershey PA Information Science Pub 2007 pp 146158.

${ }^{16}$ G C Bowker \& S L Star G

${ }_{17}$ M Christie p 8.

18 A Agrawal Dismantling the Divide Between Indigenous and Scientific Knowledge Development and Change 261998 pp 413-439.

${ }^{19} \mathrm{H}$ Verran M Christie B Anbins-King T van Weeren \& W Yunupingu Designing digital knowledge management tools with Aboriginal Australians Digital Creativity 18(3) 2007 pp 129-142.

20 Ibid p 132.

${ }^{21}$ A Mathes Folksonomies - Cooperative Classification and Communication Through Shared Metadata 2004 at http://www. adammathes.com/academic/computer-mediatedcommunication/folksonomies.html

22 M Guy \& E Tonkin Folksonomies: Tidying up Tags? D-Lib Magazine 12(1) 2006.

${ }^{23}$ A Agrawal Indigenous knowledge and the politics of classification. International Social Science Journal 54(3) 2002 p 294.

${ }^{24}$ Hunter $p 119$.

25 FileMaker Inc FileMaker and Microsoft Access: A Criteria-Based Comparison 2007 from http://www.filemaker.com/downloads/pdf/comparison_fm_access.pdf 